\title{
Avaliação da proliferação celular como indicador prognóstico para mastocitomas cutâneos caninos ${ }^{1}$
}

\author{
Ricardo De F. Strefezzi ${ }^{2 *}$ Silvia Regina Kleeb³, José Guilherme Xavier ${ }^{3,4}$ \\ e José Luiz Catão Dias ${ }^{5}$
}

\begin{abstract}
Strefezzi R.F., Kleeb S.R., Xavier J.G. \& Catão-Dias J.L. 2010. [Evaluation of cellular proliferation as prognostic indicator for canine cutaneous mast cell tumors.] Avaliação da proliferação celular como indicador prognóstico para mastocitomas cutâneos caninos. Pesquisa Veterinária Brasileira 30(7):559-565. Departamento de Zootecnia, Faculdade de Zootecnia e Engenharia de Alimentos, Universidade de São Paulo, Av. Duque de Caxias Norte 225, Pirassununga, SP 13635-900, Brazil. E-mail: rstrefezzi@ usp.br

This study evaluated the prognostic value of cell proliferation markers for canine cutaneous mast cell tumor cases. Twenty-three cases were analyzed with regard to immunohistochemical expression of Ki67 and Proliferating Cell Nuclear Antigen (PCNA), and were clinically followed up. Ki67 expression was related to the traditional histopathological grading $(p=0.0418 ; p<0.05$ between grades I and III), and was a reliable indicator of post-surgical survival $(p=0.0089)$. PCNA immunoexpression did not show statistically significant values in the prediction of disease-related mortality and survival, although it is correlated to Ki67 expression. These results confirm that information about tumoral proliferative activity through Ki67 immunohistochemical detection can improve canine cutaneous mast cell tumor grading with regard to malignancy.
\end{abstract}

INDEX TERMS: Mast cell tumor, PCNA, Ki67, proliferation markers, prognostic markers.

RESUMO.- Este estudo teve como objetivo avaliar o valor prognóstico de marcadores de proliferação celular em casos de mastocitomas cutâneos caninos. Vinte e três casos foram analisados quanto à expressão imuno-histoquímica de Ki67 e do Antígeno Nuclear de Proliferação Celular (PCNA), sendo subsequentemente acompanhados clinicamente. Observou-se que a expressão de Ki67 mantém rela-

\footnotetext{
${ }^{1}$ Recebido em 31 de outubro de 2009.

Aceito para publicação em 8 de fevereiro de 2010.

Parte da Tese de Doutorado do primeiro autor, apresentada ao Departamento de Patologia, Faculdade de Medicina Veterinária e Zootecnia, Universidade de São Paulo.

${ }^{2}$ Departamento de Zootecnia, Faculdade de Zootecnia e Engenharia de Alimentos, Universidade de São Paulo, Av. Duque de Caxias Norte 225, Pirassununga, SP 13635-900, Brazil. E-mail: rstrefezzi@usp.br

${ }^{3}$ Docente do Curso de Medicina Veterinária, Universidade Metodista de São Paulo (Umesp), Rua Dom Jaime de Barros Câmara 1000, São Bernardo do Campo, SP 09895-400, Brasil.

4 Docente do Curso de Medicina Veterinária, Universidade Paulista (Unip), Rua Dr. Bacelar 1212, São Paulo, SP 04026-002, Brasil.

${ }^{5}$ Departamento de Patologia, Faculdade de Medicina Veterinária e Zootecnia, Universidade de São Paulo (USP), Av. Prof. Dr. Orlando Marques de Paiva 87, São Paulo, SP 05508-270.
}

ção negativa com a tradicional graduação histopatológica $(p=0,0418 ; p<0,05$ entre os graus I e III), sendo um indicador confiável para o tempo de sobrevida pós-cirúrgica $(p=0,0089)$. A imunoexpressão de PCNA, apesar de estar correlacionada à marcação por Ki67, não apresentou valores estatisticamente significantes na predição da mortalidade em função da doença e do tempo de sobrevida pós-cirúrgico. Os resultados obtidos confirmam que informações sobre a atividade proliferativa tumoral pela detecção imunohistoquímica de Ki67 podem incrementar a classificação de mastocitomas cutâneos caninos quanto à malignidade.

TERMOS DE INDEXAÇÃO: Mastocitoma, PCNA, Ki67, marcadores de proliferação, marcadores de prognóstico.

\section{INTRODUÇÃO}

Os mastocitomas são os tumores de pele mais comuns em cães, chegando a representar até $27 \%$ de todos os neoplasmas malignos cutâneos. Não há evidência de predisposição sexual e sua incidência aumenta de acordo com a idade, com média dos cães afetados em torno de 8 anos e meio. As raças mais acometidas são as que possuem a Bulldog como ancestral, principalmente as Boxer, Boston 
Terrier e Bulldog Inglês, além da Labrador, Golden Retriever e Shar Pei (Hottendorf \& Nielsen 1967, Bostock 1973, Hess 1977, Patnaik et al. 1984, Yager \& Scott 1993, Simoes et al. 1994, Rogers 1996, Ginn et al. 2000, Jaffe et al. 2000, Strefezzi et al. 2003, London \& Séguin 2003, Misdorp 2004).

Os mastocitomas cutâneos podem surgir da derme ou do tecido conjuntivo subcutâneo e apresentam características macroscópicas diversas, fato que dificulta ou impossibilita especulações sobre o comportamento desse tipo de neoplasia quando nos baseamos apenas nestas características (London \& Séguin 2003, Strefezzi et al. 2003). Estes tumores são considerados por clínicos e cirurgiões como neoplasmas potencialmente malignos e, devido a seu comportamento extremamente variável, têm provocado frustrações terapêuticas (Macy 1985, O’Keefe 1990, Yager \& Scott 1993, Simoes et al. 1994).

Devido à dificuldade de previsão do comportamento biológico e, consequentemente, de definição dos tratamentos instituídos, a busca por indicadores prognósticos mais precisos para o mastocitoma vem se intensificando e se diversificando nas últimas décadas. A graduação histopatológica tem sido o método de eleição na tentativa de prever o comportamento biológico deste neoplasma, dividindo os tumores em três graus crescentes de malignidade (Patnaik et al. 1984). Entretanto, conforme observado por diversos autores, tal procedimento baseia-se em parâmetros subjetivos como índice mitótico, quantidade de grânulos citoplasmáticos e grau de anisocitose, provocando variações intra e interobservador consideráveis, principalmente no que se refere aos tumores de diferenciação intermediária (Yager \& Scott 1993, Simoes et al. 1994, Strefezzi et al. 2003, Misdorp 2004, Kiupel et al. 2005, Maiolino et al. 2005, Northrup et al. 2005, Pinczowski et al. 2008).

A mensuração da atividade proliferativa pode acrescentar informações importantes para a formulação de um prognóstico mais confiável identificando, por meio de marcações histo e imuno-histoquímicas ou da contagem de mitoses, a fração de células em proliferação (Ellis \& Whitehead 1981, Quinn \& Wright 1990, Abadie et al. 1999). Entre esses métodos, merecem destaque a contagem de mitoses (Romansik et al. 2007), a determinação da frequência de Regiões Organizadoras Nucleolares com afinidade pela prata (AgNOR) (Bostock et al. 1989, Simoes et al. 1994, Kravis et al. 1996), a contagem de células positivas para o Antígeno Nuclear de Proliferação Celular (PCNA) (Simoes et al. 1994, Abadie et al. 1999) e a contagem de células positivas para Ki67 (Abadie et al. 1999, Sakai et al. 2002).

O PCNA é uma proteína não-histona necessária à síntese e reparo de DNA, detectada desde a fase G1 até a transição $\mathrm{G} 2$ a $\mathrm{M}$, atingindo seu máximo na fase $\mathrm{S}$ do ciclo celular (Madewell 2001). Está ausente na fase G0, mas, diferentemente do Ki67, sua expressão varia muito nas fases G1, S, G2 e M. Pode ser induzida por dano ao DNA e, ainda, possui meia-vida longa, fazendo com que as frações proliferativas sejam, muitas vezes, super-representadas (Abadie et al. 1999, Madewell 2001).
Atualmente, a marcação imuno-histoquímica de Ki67, antígeno possivelmente relacionado ao RNA nucleolar, é a ferramenta mais utilizada para avaliação da atividade proliferativa de uma população celular por associar características desejáveis para este tipo de marcador, como a expressão durante praticamente todo o ciclo celular, de início de G1 a $\mathrm{M}$, e por possuir meia-vida curta, sendo degradado em aproximadamente uma hora após a mitose. Sendo assim, mesmo marcações tênues podem ser consideradas positivas, dispensando os pontos de corte, geralmente utilizados para a avaliação do PCNA (Quinn \& Wright 1990, Abadie et al. 1999, Melo \& Alves 1999, Madewell 2001, Scase et al. 2006).

Com relação aos mastocitomas cutâneos, a avaliação da atividade proliferativa revelou que o índice de PCNA foi maior nos cães que morreram em função do mastocitoma do que nos que sobreviveram, mas, apesar disso, não foi demonstrada diferença estatisticamente significante entre os graus histopatológicos (Abadie et al. 1999, Scase et al. 2006). A contagem de células positivas para PCNA foi considerada um indicador de sobrevida confiável por Simoes et al. (1994), porém não apresentou valor prognóstico nos estudos realizados por Scase et al. (2006) e Webster et al. (2007). Já para o Ki67 houve diferença significante entre os graus histopatológicos e o mesmo foi considerado útil como indicador prognóstico independente da graduação histológica, principalmente na subdivisão de mastocitomas de grau II quanto à agressividade (Abadie et al. 1999, Sakai et al. 2002, Scase et al. 2006). Atualmente, recomenda-se a utilização de Ki67, em associação à avaliação da expressão de $c-K I T$ e a contagem de AgNORs como métodos rotineiros para indicação do prognóstico em mastocitomas (Webster et al. 2007)

Frente aos resultados conflitantes observados na utilização de PCNA como indicador prognóstico para mastocitomas cutâneos caninos, bem como às vantagens relatadas para a avaliação da expressão de Ki67, o objetivo deste trabalho foi verificar a eficiência de tais marcações como métodos de previsão do tempo de sobrevida e da mortalidade em função da doença em cães tratados cirurgicamente, comparando-as à graduação histopatológica tradicional (Patnaik et al. 1984).

\section{MATERIAL E MÉTODOS}

Foram analisados 27 mastocitomas cutâneos caninos, obtidos a partir de 23 casos atendidos no ambulatório cirúrgico do Hospital Veterinário da Universidade de São Paulo (HOVETUSP), Departamento de Cirurgia (VCI) da Faculdade de Medicina Veterinária e Zootecnia da Universidade de São Paulo (FMVZ-USP), e nos Hospitais Veterinários da Universidade Bandeirante de São Paulo (UNIBAN) e Universidade Metodista de São Paulo (UMESP). A evolução clínica dos casos foi avaliada por meio de análise de prontuários hospitalares e entrevistas com proprietários e médicos veterinários responsáveis. Destes, $12(52,2 \%)$ eram machos e 11 fêmeas $(47,8 \%)$. A raça mais frequente foi a Boxer $(26,1 \%, 6 / 23)$ seguida das raças Poodle $(17,4 \%, 4 / 23)$, Dachshund e Fila Brasileiro (8,7\%, 2/23 cada). Cinco cães $(21,8 \%)$ eram sem raça definida. Os demais eram das raças Doberman Pinscher, Dogue Alemão, Labrador e 
Pinscher. Quatro cães apresentavam lesões múltiplas (17,4\%, 4/23), dois da raça Boxer, um Doberman e um Poodle. A idade média dos cães foi de oito anos e meio.

Os critérios para seleção dos casos incluídos neste estudo foram: (1) excisão cirúrgica completa dos tumores como forma de tratamento; (2) disponibilidade de histórico clínico completo de no mínimo 120 dias; (3) disponibilidade e qualidade das amostras histológicas para análise.

As peças cirúrgicas foram fixadas em solução de formaldeído a $10 \%$ por 48 horas. Delas foram retirados fragmentos e estes encaminhados ao Laboratório de Patologia Celular e Molecular do Hospital Sírio-Libanês, onde sofreram processamento histológico de acordo com as técnicas rotineiras de inclusão em parafina. Cortes de aproximadamente $5 \mu \mathrm{m}$ de espessura foram obtidos e corados pela técnica da Hematoxilina e Eosina (Prophet et al. 1992). A coloração especial de Azul de Toluidina (Prophet et al. 1992) e/ou a marcação positiva com anticorpos anti-KIT foram realizadas para a confirmação do diagnóstico de mastocitoma.

O processamento imuno-histoquímico baseou-se no método descrito por Hsu et al. (1981). Os cortes histológicos, aderidos a lâminas silanizadas, foram desparafinados em estufa e, em seguida, reidratados em álcool graduado e água destilada. A recuperação de antígenos foi realizada por aquecimento dos cortes histológicos em solução tampão. Para o anticorpo antiPCNA foi utilizado tampão citrato com pH 6,0 e para o anti-Ki67 o tampão foi EDTA, com pH 9,0. Após bloqueio de peroxidase endógena com solução de peróxido de hidrogênio a $3 \%$, os cortes histológicos foram submetidos às incubações com anticorpos primários para Ki67 ${ }^{6}$ (1:50) e $\mathrm{PCNA}^{7}$ (1:100) por 12 horas (over night) a $8^{\circ} \mathrm{C}$, anticorpo secundário biotinilado ${ }^{8}$ e, finalmente, com complexo estreptavidina-peroxidase ${ }^{9}$. A revelação foi obtida com solução contendo diaminobenzidina e a contracoloração com Hematoxilina de Harris (Hsu et al. 1981). Os controles positivos utilizados foram linfonodos caninos.

Os mastocitomas foram classificados em graus I, II ou III, de acordo com o nível decrescente de diferenciação celular. Os critérios de graduação adotados foram aqueles estabelecidos por Patnaik et al. (1984). Todos os tumores foram avaliados histopatologicamente por quatro observadores, de forma independente e sem conhecimento de informações clínicas dos cães, inclusive sobre lesões pertencentes a um mesmo cão. Em casos de variação interobservador (48,1\% das lesões, 13/ 27), o grau histopatológico considerado como diagnóstico final foi decidido por consenso.

As avaliações dos índices de proliferação celular com Ki67 e PCNA foram realizadas através da determinação da porcentagem de núcleos positivos em um mínimo de 1.000 núcleos por caso, em campos de alta frequência de marcação (hot spots) à objetiva de 40 aumentos. Núcleos positivos e negativos foram quantificados manualmente com auxílio do software Image ProPlus $®$, através da ferramenta Manual Tag. Foram

\footnotetext{
6 Monoclonal, murino, anti-Ki67 humana, clone MIB-1, Dako Cytomation, Inc., Carpinteria, CA, USA.

${ }^{7}$ Monoclonal, murino, anti-PCNA de rato, clone PC-10, Dako Cytomation, Inc., Carpinteria, CA, USA.

${ }^{8}$ Biotinylated Link Universal, Kit LSAB® + System-HRP, Ref. K0690, Dako Cytomation, Inc., Carpinteria, CA, USA.

${ }^{9}$ Streptavidin-HRP, Kit LSAB® + System-HRP, Ref. 0690, Dako Cytomation, Inc., Carpinteria, CA, USA.

10 Versão 4.02 for Windows, Graphpad Software, Inc., San Diego, CA, USA.
}

considerados positivos todos os núcleos com evidências de marcação, mesmo que fraca, conforme realizado por outros (Simoes et al. 1994, Abadie et al. 1999, Sakai et al. 2002, Scase et al. 2006). Para casos com múltiplas lesões, foram considerados os valores mais elevados dos escores de PCNA e KI67.

Os resultados obtidos nas marcações para a PCNA e Ki67 foram comparados aos graus histopatológicos por meio de ANOVA/Kruskal-Wallis, seguida de teste de múltiplas comparações de Dunn. Um ponto de corte para os valores de PCNA e Ki67 foi determinado por meio de uma curva ROC, para subdivisão das marcações em escores baixos (denominados PCNA-1 e Ki67-1) e altos (denominados PCNA-2 e Ki67-2). As correlações entre os indicadores prognósticos e o tempo de sobrevida foram analisadas pelo Teste de Spearman.

A graduação histopatológica e os escores de PCNA e Ki67 foram analisados individualmente com relação à mortalidade em consequência da doença pelos testes de Chi-quadrado para tendências e exato de Fisher. A análise de sobrevida foi realizada pelo método de Kaplan-Meier seguido de logrank test. Foram considerados censurados na análise de sobrevida os cães com histórico clínico interrompido e os que morreram ou sofreram eutanásia por motivos não relacionados ao mastocitoma cutâneo.

Os dados foram analisados com auxílio do software GraphPad Prism ${ }^{10}$. O nível de significância foi estabelecido em $5 \%$.

\section{RESULTADOS}

A graduação histológica das lesões mostrou que $21,7 \%$ de casos pertenciam ao grau I (5/23), 52,2\% ao grau II (12/23) e $26,1 \%$ ao $(6 / 23)$ grau III. Durante o período de acompanhamento clínico total, 73,9\% (17/23) dos cães morreram, sendo que sete dentre os óbitos foram relacionados ao tumor $(30,4 \%, 7 / 23)$. Nenhum cão com mastocitoma de grau I morreu em função do neoplasma, enquanto $15,4 \%$ dos de grau II (2/13) e $83,4 \%$ dos de grau III (5/6) tiveram suas mortes relacionadas à doença $\left(\chi^{2}=9,505 ; p=0,0020\right)$ (Quadro 1$)$. $O$ tempo de sobrevida pós-cirúrgica dos cães analisados variou de 3 a 2.670 dias, com média de 894 dias. Entre os cães que morreram em função dos mastocitomas, o tempo médio de acompanhamento pós-cirúrgico foi 273 dias.

A análise de sobrevida em função dos graus histopatológicos revelou diferenças significantes entre os grupos $(p<0,0001)$. Dentre os casos de grau III, nenhum cão so-

Quadro 1. Distribuição das taxas de mortalidade, relacionadas ou não aos mastocitomas, em função da graduação histopatológica proposta por Patnaik et al. (1984). Porcentagens em relação aos totais de casos para cada grau histopatológico

\begin{tabular}{lccc}
\hline & $\begin{array}{c}\text { Morte } \\
\text { relacionada } \\
\text { ao tumor }\end{array}$ & $\begin{array}{c}\text { Morte não- } \\
\text { relacionada } \\
\text { ao tumor }\end{array}$ & $\begin{array}{c}\text { Vivos ao final } \\
\text { do estudo }\end{array}$ \\
\hline Grau I $(n=5)$ & $0(0,0 \%)$ & $4(80,0 \%)$ & $1(20,0 \%)$ \\
Grau II $(n=12)$ & $2(16,6 \%)$ & $6(50,0 \%)$ & $4(33,4 \%)$ \\
Grau III $(n=6)$ & $5(83,4 \%)$ & $0(0,0 \%)$ & $1(16,6 \%)$ \\
Total $(n=23)$ & $7(30,4 \%)$ & $10(43,5 \%)$ & $6(26,1 \%)$ \\
\hline
\end{tabular}

Teste de Chi-quadrado para tendências; $\chi^{2}=9,505 ; p=0,0020 ; n=$ número de casos. 


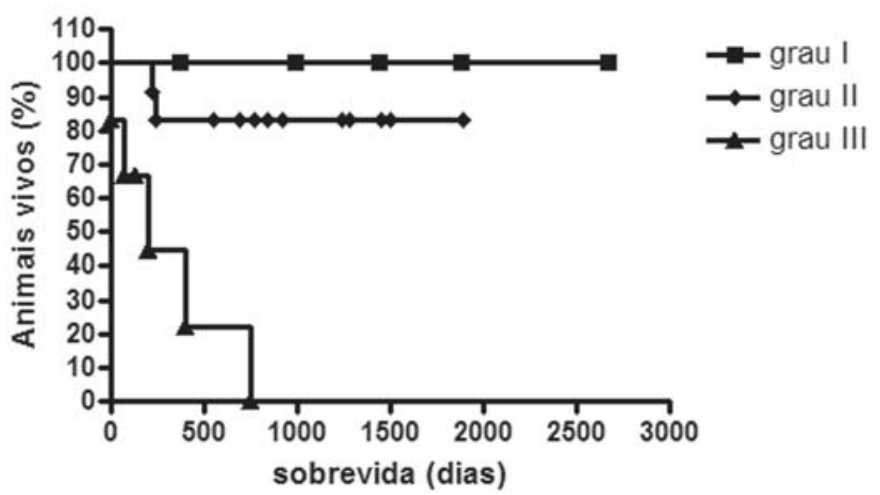

Fig.1. Curva de sobrevida dos casos estudados em função da graduação histopatológica baseada na proposta de Patnaik et al. (1984) $(p<0,0001$, logrank test). Pontos = dados censurados pela análise de Kaplan-Meier.

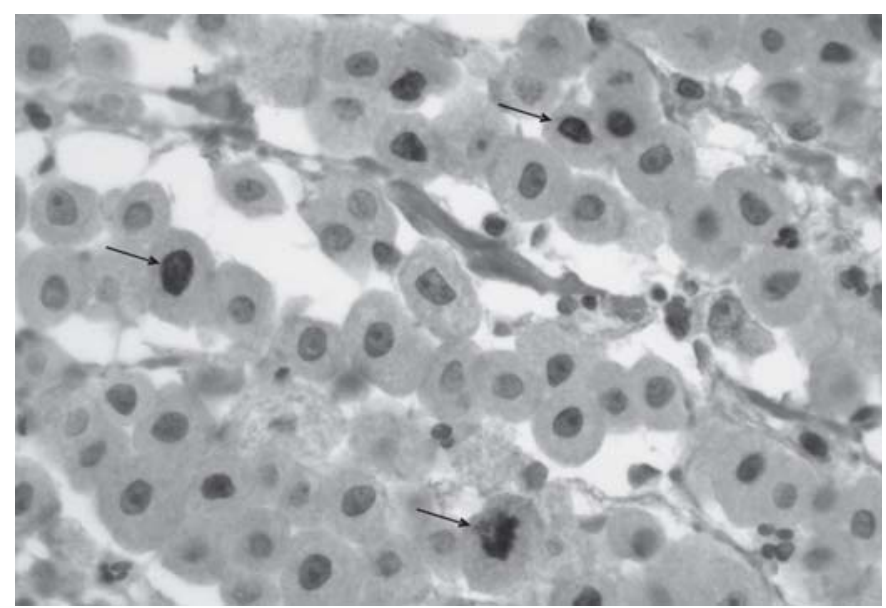

Fig.2. Mastocitoma cutâneo representando imunorreatividade nuclear para PCNA (setas). Kit-LSAB®/diaminobenzidina, contracoloração com Hematoxilina de Harris, obj.40x.

breviveu por mais de 751 dias, com mediana de sobrevida de 207 dias (média de 261 dias). Por outro lado, nenhum dos cães com mastocitoma de grau I teve sua morte relacionada ao neoplasma (média de sobrevida de 1.473 dias) e $83,3 \%$ dos cães com mastocitoma de grau II atingiram sobrevida de até 1.898 dias pela análise de Kaplan-Meier (média de 969 dias) (Fig.1).

A porcentagem média de núcleos positivos para PCNA (Fig.2) foi de $22,8 \pm 19,1 \%$, sendo $28,2 \pm 23,2 \%$ entre os que morreram em função do tumor e $20,5 \pm 17,3 \%$ entre os que não tiveram suas mortes relacionadas com o neoplasma. Não foi detectada correlação entre as porcentagens de PCNA e a sobrevida dos casos analisados $(p=0,1003)$. Para os cães com escores PCNA-1, a taxa de mortalidade em função dos mastocitomas foi de 23,1\% (3/13) contra 40\% (4/ 10) para os casos de escore PCNA-2 ( $p=0,0657)$ (Quadro 2). Quando comparados os escores de PCNA entre os graus histopatológicos, não foram encontradas diferenças estatisticamente significativas $(p=0,1079)$.

Em seguida, os casos foram divididos em dois grupos: escores baixos de PCNA (PCNA-1) e escores altos (PCNA-
Quadro 2. Distribuição das taxas de mortalidade, relacionadas ou não aos mastocitomas, em função dos escores de PCNA. Porcentagens em relação aos totais de casos de cada padrão de marcação

\begin{tabular}{cccc}
\hline & $\begin{array}{c}\text { Morte } \\
\text { relacionada } \\
\text { ao tumor }\end{array}$ & $\begin{array}{c}\text { Morte não- } \\
\text { relacionada } \\
\text { ao tumor }\end{array}$ & $\begin{array}{c}\text { Vivos ao final } \\
\text { do estudo }\end{array}$ \\
\hline $\begin{array}{c}\text { PCNA-1 } \\
(<24 \%)(n=13)\end{array}$ & $3(23,1 \%)$ & $7(53,8 \%)$ & $3(23,1 \%)$ \\
$\begin{array}{c}\text { PCNA-2 } \\
(\geq 24 \%)(n=10)\end{array}$ & $4(40,0 \%)$ & $3(30,0 \%)$ & $3(30,0 \%)$ \\
Total $(n=23)$ & $7(30,4 \%)$ & $10(43,5 \%)$ & $6(26,1 \%)$
\end{tabular}

Teste exato de Fisher, $\mathrm{p}=0,6500 ; \mathrm{n}=$ número de casos.

2). O ponto de corte foi determinado por uma curva ROC: PCNA-1, com menos de $24 \%$ de células positivas; e PCNA2 , com positividade maior ou igual a $24 \%$ (sensibilidade = $57,1 \%$; especificidade $=62,5 \%$ ). A análise de sobrevida dos grupos não revelou diferenças estatisticamente significativas ( $p=0,2023$ ) (Fig.3).

A porcentagem média de núcleos positivos para Ki67 (Fig.4) foi de 6,6 $\pm 6,3 \%$, sendo $9,4 \pm 6,1 \%$ entre os que

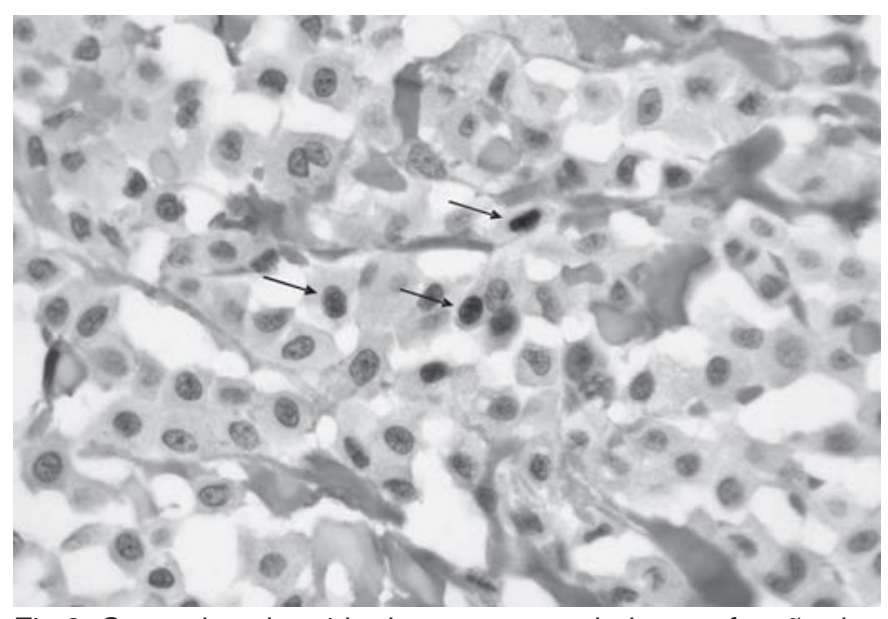

Fig.3. Curva de sobrevida dos casos estudados em função dos escores de PCNA: PCNA-1 (positividade <24\%) ou PCNA2 (positividade $\geq 24 \%$ ) ( $p=0,2023$, logrank test; pontos = dados censurados pela análise de Kaplan-Meier).

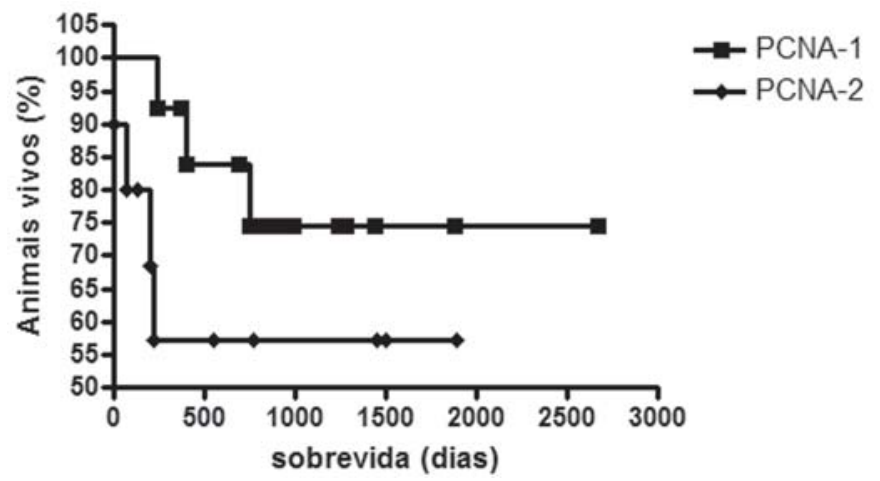

Fig.4. Mastocitoma cutâneo representando imunorreatividade nuclear para Ki67 (setas). Kit-LSAB®/diaminobenzidina, contracoloração com Hematoxilina de Harris, obj.40x. 
Quadro 3. Distribuição das taxas de mortalidade, relacionadas ou não aos mastocitomas, em função dos escores de Ki67. Porcentagens em relação aos totais de casos de cada padrão de marcação

\begin{tabular}{cccc}
\hline & $\begin{array}{c}\text { Morte } \\
\text { relacionada } \\
\text { ao tumor }\end{array}$ & $\begin{array}{c}\text { Morte não- } \\
\text { relacionada } \\
\text { ao tumor }\end{array}$ & $\begin{array}{c}\text { Vivos ao final } \\
\text { do estudo }\end{array}$ \\
\hline $\begin{array}{c}\text { Ki67-1 } \\
(<7,0 \%)(n=14) \\
\mathrm{Ki} 67-2\end{array}$ & $2(14,3 \%)$ & $8(57,1 \%)$ & $4(28,6 \%)$ \\
$(\geq 7,0 \%)(n=9)$ & $5(55,6 \%)$ & $2(22,2 \%)$ & $2(22,2 \%)$ \\
Total $(n=23)$ & $7(30,4 \%)$ & $10(43,5 \%)$ & $6(26,1 \%)$ \\
\hline
\end{tabular}

Teste exato de Fisher, $\mathrm{p}=0,0657 ; \mathrm{n}=$ número de casos.

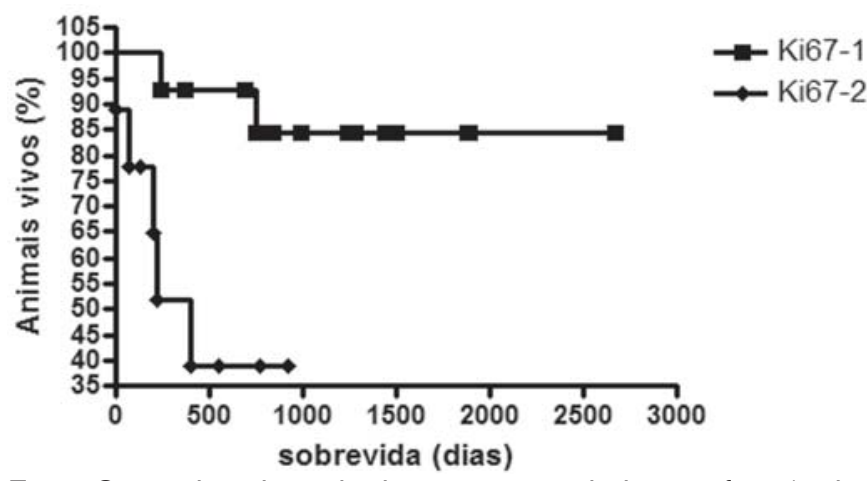

Fig.5. Curva de sobrevida dos casos estudados em função dos escores de Ki67, Ki67-1 (<7,0\%) ou Ki67-2 ( $\geq 7,0 \%)$ $(p=0,0089$, logrank test; pontos $=$ dados censurados pela análise de Kaplan-Meier).

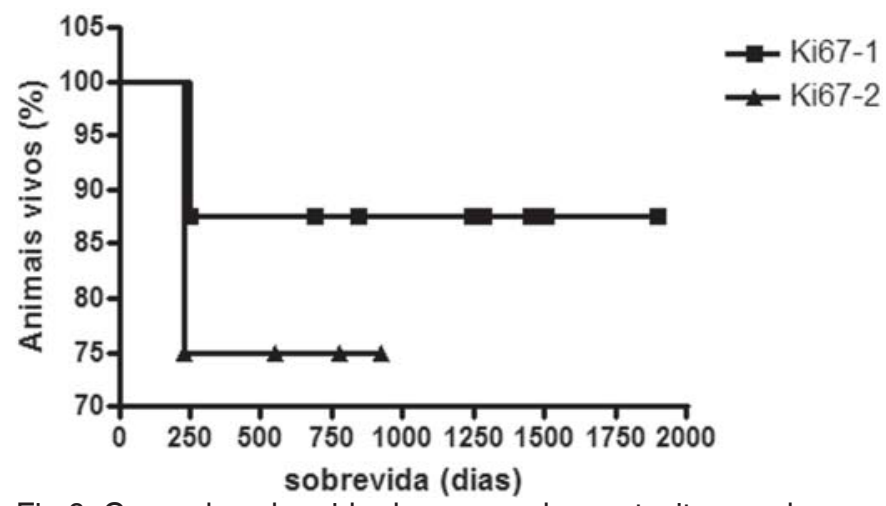

Fig.6. Curva de sobrevida dos casos de mastocitomas de grau Il estudados em função dos escores de Ki67, Ki67-1 (<7,0\%) ou Ki67-2 ( $\geq 7,0 \%)$, $(p=0,5436$, logrank test; pontos = dados censurados pela análise de Kaplan-Meier).

morreram em função do tumor e 5,5 $\pm 6,2 \%$ entre os que não tiveram seus óbitos relacionados com o neoplasma. Houve correlação negativa entre os escores de Ki67 e a sobrevida $(r=-0,5432$ e $p=0,0074)$ e positiva quando comparados aos escores de PCNA ( $r=0,7470, p=<0,0001)$. A comparação dos escores de Ki67 quando divididos com base nos graus histopatológicos revelou diferenças estatisticamente significativas $(p=0,0418 ; p<0,05$ entre graus I e III).

De modo semelhante ao realizado para PCNA, os casos foram divididos em dois grupos: Ki67-1, com menos de 7,0\% de células positivas; e Ki67-2, com positividade maior ou igual a $7,0 \%$ (sensibilidade $=71,4 \%$; especificidade $=75,0 \%$ ). Para os cães com escores Ki67-1, a taxa de mortalidade em função dos mastocitomas foi de 14,3\% (2/14) contra 55,6\% (5/9) para os casos de escore Ki67-2 $(p=0,0657)$ (Quadro 3). A sobrevida de cães do grupo Ki671 foi significantemente maior que a do grupo Ki67-2 ( $p=$ 0,0089) (Fig.5). Porém, o índice não foi capaz de distinguir entre mastocitomas de grau II mais ou menos agressivos $(p=0,5436)$ (Fig.6).

\section{DISCUSSÃO E CONCLUSÕES}

A tradicional graduação histológica proposta por Patnaik et al. (1984) tem sido utilizada como padrão-ouro de classificação de mastocitomas cutâneos caninos por apresentar resultados expressivos na previsão do comportamento biológico deste neoplasma (Macy 1985, Strefezzi et al. 2003, Misdorp 2004, Maiolino et al. 2005, Kiupel et al. 2005). Entretanto, está alicerçada em parâmetros histomorfológicos geralmente subjetivos, o que conduz a variações inter e intraobservadores (Strefezzi et al. 2003, Kiupel et al. 2005, Northrup et al. 2005, Pinczowski et al. 2008).

A avaliação de situações limítrofes entre os diferentes graus permanece desafiadora, pois parte dos tumores de grau II comporta-se como mastocitomas de grau I, enquanto outros como de grau III. Isto significa que alguns tumores têm seus potenciais de agressividade subestimados, enquanto outros recebem tratamentos potencialmente tóxicos de maneira desnecessária. Northrup et al. (2005) sugeriram que uma nova classificação de mastocitomas em tumores de alto ou baixo-grau seria mais consistente e de maior significado prognóstico.

Simoes et al. (1994) compararam o valor da graduação histopatológica proposta por Patnaik et al. (1984) e a fração marcada pelo PCNA como indicadores prognósticos. Para estes autores, a fração marcada por PCNA, em cinco campos de 40 aumentos, foi um bom indicador prognóstico quanto à sobrevida e recidiva. Para Abadie et al. (1999) e Scase et al. (2006), o índice de PCNA foi maior nos cães que morreram em função do mastocitoma do que nos que sobreviveram mas, apesar disso, não foi demonstrada diferença estatisticamente significante quando os casos foram agrupados com base nos graus histopatológicos e no tempo de sobrevida.

Apesar de estar correlacionado com a marcação para Ki67, acredita-se que a longa meia-vida de PCNA possa superestimar a atividade proliferativa no tumor, sendo então necessários pontos de corte para sua avaliação (Quinn \& Wright 1990, Abadie et al. 1999, Melo \& Alves 1999, Madewell 2001, Scase et al. 2006). Certamente, tais limites ou pontos de corte, referentes a intensidades mínimas de marcação imuno-histoquímica para positividade, adicionariam subjetividade e, consequentemente, maiores variações intra a interobservador na contagem de células positivas para PCNA. Por este motivo, optamos por considerar positivas quaisquer marcações, mesmo que tênues, assim como é realizado para o Ki67. 
Os resultados obtidos neste trabalho em relação ao PCNA não são animadores. Não foram detectadas relações significantes entre PCNA e graduação histopatológica e, quando estabelecidos valores de corte para divisão em dois grupos, as curvas de sobrevida não foram estatisticamente diferentes, corroborando resultados relatados por outros autores (Abadie et al. 1999, Scase et al. 2006).

Observamos correlação negativa entre Ki67 e sobrevida, ou seja, quanto maior a porcentagem de células positivas Ki67, menor a sobrevida pós-cirúrgica dos cães. Foram detectadas diferenças estatisticamente significantes entre os graus I e III com relação à marcação para Ki67. O agrupamento em escores menores ou maiores que 7,0\% (Ki67-1 e Ki67-2) resultou em indicadores confiáveis para a sobrevida pós-cirúrgica de cães com mastocitomas cutâneos. Estes resultados corroboram outros relatos sobre as potencialidades do Ki67 como indicador prognóstico para mastocitomas (Abadie et al. 1999, Sakai et al. 2002, Scase et al. 2006, Webster et al. 2007).

Scase et al. (2006) afirmaram que a avaliação de Ki67 pode ser útil, principalmente, na subdivisão de mastocitomas de grau II quanto à agressividade. Contudo, quando analisamos somente mastocitomas de grau II com base nos escores de Ki67, não foram detectadas diferenças significantes nos períodos de sobrevida. Estas diferenças podem estar relacionadas com o número de cães analisados, pois neste trabalho foram estudados 12 casos, enquanto Scase et al. (2006) analisaram 86 e Webster et al. (2007) analisaram 41. É possível que, se analisássemos uma amostra maior, talvez pudéssemos identificar dois grupos distintos entre os tumores de grau II em relação às porcentagens de células positivas para Ki67.

Apesar de questionada por muitos (Macy 1985, Abadie et al. 1999, Strefezzi et al. 2003, Kiupel et al. 2004, Misdorp 2004, Northrup et al. 2005, Scase et al. 2006, Romansik et al. 2007, Webster et al. 2007), a tradicional graduação histopatológica de Patnaik et al. (1984) é, ainda, o método que obtém os melhores resultados na previsão do comportamento de mastocitomas cutâneos caninos. Este estudo reafirma que a graduação histopatológica é um excelente indicador para mortalidade relacionada ao tumor, que aumenta conforme o grau histopatológico: $0 \%$ para os de grau I, $16,6 \%$ para os de grau II e 83,4\% para os de grau III.

Observa-se que a mortalidade para os mastocitomas de diferenciação intermediária se mantém baixa, mais próxima da dos de grau I do que da mortalidade dos de grau III. Esta distribuição corrobora observações de Séguin et al. (2001) e Weisse et al. (2002), que descartam necessidade de quimioterapia para os mastocitomas de grau II em casos de ressecção cirúrgica completa. Como indicador para tempo de sobrevida pós-cirúrgica, a graduação histológica também se mostrou melhor indicador entre os parâmetros testados sendo que, mesmo com um pequeno número de casos analisados $(n=23)$, os resultados obtidos por este método continuam bastante expressivos.

A maioria das críticas à classificação histomorfológica se dá em relação à subjetividade na classificação dos tu- mores, pela somatória de critérios histomorfológicos de difícil padronização/mensuração, gerando variações intra e interobservador em grande parte dos casos (Yager \& Scott 1993, Simoes et al. 1994, Strefezzi et al. 2003, Misdorp 2004, Kiupel et al. 2005, Maiolino et al. 2005, Northrup et al. 2005, Pinczowski et al. 2008). Com a finalidade de reduzir tais variações, determinamos que o grau histopatológico final fosse obtido por consenso entre quatro observadores com experiência em histopatologia. Acreditamos, ainda, que o método possa ser aperfeiçoado, dividindo-se os neoplasmas em apenas dois graus, conforme sugerido por Northrup et al. (2005). Estabelecendo critérios mais objetivos, tais como a mensuração de parâmetros morfométricos nucleares, contagem de mitoses, ou outras características peculiares ao tumor, adicionaríamos parâmetros mais reprodutíveis e precisos ao método proposto por Patnaik et al. (1984).

Finalmente, os resultados aqui descritos reforçam a utilidade da avaliação histopatológica de mastocitomas cutâneos caninos na previsão da mortalidade e sobrevida pós-cirúrgica, e confirmam que essa previsão pode ser incrementada por informações sobre a atividade proliferativa tumoral pela detecção imuno-histoquímica de Ki67.

Agradecimentos.- À Profa. Dra. Julia Maria Matera, Departamento de Cirurgia da FMVZ-USP, e aos médicos veterinários dos Hospitais Veterinários da Universidade Bandeirante de São Paulo (UNIBAN) e Universidade Metodista de São Paulo (UMESP) por submeterem o material cirúrgico. Ao Dr. L.H. Câmara Lopes, Laboratório de Patologia Celular e Molecular do Hospital Sírio-Libanês, pelo processamento imuno-histoquímico; à Fundação de Amparo à Pesquisa do Estado de São Paulo (FAPESP), pelo apoio financeiro (Processos 05/50407-3, 97/09459-7 e 96/4903-8)

\section{REFERÊNCIAS}

Abadie J.J., Amardeilh M.A. \& Delverdier M.E. 1999. Immunohistochemical detection of proliferating cell nuclear antigen and Ki-67 in mast cell tumors from dogs. J. Am. Vet. Med. Assoc. 215:1629-1634.

Bostock D.E. 1973. The prognosis following surgical removal of mastocytomas in dogs. J. Small Anim. Pract. 14:27-40.

Bostock D.E., Crocker J., Harris K. \& Smith P. 1989. Nucleolar organizer regions as indicators of post surgical prognosis in canine spontaneous mast cell tumors. Brit. J. Cancer 59:915-918.

Ellis P.S.J. \& Whitehead R. 1981. Mitosis counting: A need to reappraisal. Hum. Pathol. 12:1-4.

Ginn P.E., Fox L.E., Brower J.C., Gaskin A., Kurzman I.D. \& Kubilis P.S. 2000. Immunohistochemical detection of p53 tumor-suppressor protein is a poor indicator of prognosis for canine cutaneous mast cell tumors. Vet. Pathol. 37:33-39

Hess P.W. 1977. Canine mast cell tumors. Vet. Clin. North Am., Small Anim. Pract. 7:133-143.

Hottendorf G.H. \& Nielsen S.W. 1967. Pathologic survey of 300 extirpated canine mastocytomas. Zentralbl. Veterinärmed A 14:272-281.

Hsu M.K., Raine L. \& Fanger H. 1981. Use of avidin-biotin-peroxidase complex $(A B C)$ in immunoperoxidase techniques: A comparison between $A B C$ and unlabeled antibody (PAP) procedures. J. Histochem. Cytochem. 29:577-580.

Jaffe M.H., Hosgood G., Taylor H.W., Kerwin S.C., Hedlung C.S., Lopez M.K., Davidson J.R., Miller D.M. \& Paranjepe M. 2000. Immunohistochemical and clinical evaluation of p53 in canine cutaneous mast cell tumors. Vet. Pathol. 37:40-46. 
Kiupel M., Webster J.D., Kaneene J.B., Miller R. \& Yuzbasiyan-Gurkan V. 2004. The use of KIT and Tryptase expression patterns as prognostic tools for canine cutaneous mast cell tumors. Vet. Pathol. 41:371-377.

Kiupel M., Webster J.D., Miller R.A. \& Kaneene J.B. 2005. Impact of tumour depth, tumour location and multiple synchronous masses on the prognosis of canine cutaneous mast cell tumours. J. Vet. Med. A 52:280-286.

Kravis L.D., Vail D.M., Kisseberth W.C., Ogilvie G.K. \& Volk L.M. 1996. Frequency of argyrophilic organizer regions in fine-needle aspirates and biopsy specimens from mast cell tumors in dogs. J. Am. Vet. Med. Assoc. 209:1418-1420.

London C.A. \& Séguin B. 2003. Mast cell tumors in the dog. Vet. Clin. North Am., Small Anim. Pract. 33:473-489.

Macy D.W. 1985. Canine mast cell tumors. Vet. Clin. North Am., Small Anim. Pract. 15:783-803.

Madewell B.R. 2001. Cellular proliferation in tumors: A review of methods, interpretation, and clinical applications. J. Vet. Int. Med. 15:334-340.

Maiolino P., Cataldi M., Paciello O., Restucci B. \& De Vico G. 2005. Nucleomorphometric analysis of canine cutaneous mast cell tumours. J. Comp. Pathol. 133:209-211.

Melo E.S. \& Alves V.A.F. 1999. Marcadores de proliferação celular, p.182-194. In: Alves V.A.F., Bacchi C.E. \& Vassalo J. (Eds), Manual de Imuno-histoquímica. Sociedade Brasileira de Patologia, São Paulo.

Misdorp W. 2004. Mast cells and canine mast cell tumours: A review. Vet. Quart. 26:156-169.

Northrup N.C., Howerth E.W., Harmon B.G., Brown C.A., Carmicheal K.P., Garcia A.P., Latimer K.S., Munday J.S., Rakich P.M., Richey L.J., Stedman N.L. \& Gieger T.L. 2005. Variation among pathologists in the histologic grading of canine cutaneous mast cell tumors with uniform use of a single grading reference. J. Vet. Diagn. Invest. 17:561-564.

O'Keefe D.A. 1990. Canine mast cell tumors. Vet. Clin. North Am., Small Anim. Pract. 20:1105-1115.

Patnaik A.K., Ehler W.J. \& Macewem E.G. 1984. Canine cutaneous mast cell tumor: morphologic grading and survival time in 83 dogs. Vet. Pathol. 21:469-474.

Pinczowski P., Torres Neto R., Fabris V.E. \& Laufer-Amorim R. 2008.
Mastocitoma cutâneo canino: variação da graduação histopatológica entre patologistas. Revta Clín. Vet. 77:76-78.

Prophet E.B., Mills B., Arrington J.B. \& Sobin L.H. 1992. Laboratory methods in histotechnology. Armed Forces Institute of Pathology, Washington. 279p.

Quinn C.M. \& Wright N.A. 1990. The clinical assessment of proliferation and growth in human tumours: evaluation of methods and applications as prognostic variables. J. Pathol. 160:93-102.

Rogers K.S. 1996. Mast cell tumors: dilemmas of diagnosis and treatment. Vet. Clin. North Am., Small Anim. Pract. 26:87-102.

Romansik E.M., Reilly C.M., Kass P.H., Moore P.F. \& London C.A. 2007. Mitotic index is predictive for survival for canine cutaneous mast cell tumors. Vet. Pathol. 44:335-341.

Sakai H., Noda A., Shirai N., lidaka T., Yanai T. \& Masegi T. 2002. Proliferative activity of canine mast cell tumours evaluated by bromodeoxyuridine incorporation and Ki-67 expression. J. Comp. Pathol. 127:233-238.

Scase T.J., Edwards D., Miller J., Henley W., Smith K., Blunden A. \& Murphy S. 2006. Canine mast cell tumors: correlation of apoptosis and proliferation markers with prognosis. J. Vet. Int. Med. 20:151-158.

Séguin B., Leibman N.F., Bregazzi V.S., Ogilvie G.K., Powers B.E., Dernell W.S., Fettman M.J. \& Withdrow S.J. 2001. Clinical outcome of dogs with grade-II mast cell tumors treated with surgery alone: 55 cases (1996-1999). J. Am. Vet. Med. Assoc. 218:1120-1123.

Simoes J.P.C., Schoning P. \& Butine M. 1994. Prognosis of canine mast cell tumors: A comparison of three methods. Vet. Pathol. 31:637-647.

Strefezzi R.F., Xavier J.G. \& Catão-Dias J.L. 2003. Morphometry of canine cutaneous mast cell tumors. Vet. Pathol. 40:268-275.

Webster J.D., Yuzbasiyan-Gurkan V., Miller R., Kaneene J.B. \& Kiupel M. 2007. Cellular proliferation in canine cutaneous mast cell tumors: Associations with c-KIT and its role in prognostication. Vet. Pathol. 44:298-308.

Weisse C., Shofer F.S. \& Sorenmo K. 2002. Recurrence rates and sites for grade II canine cutaneous mast cell tumors following complete surgical excision. J. Am. Anim. Hosp. Assoc. 38:71-73.

Yager J.A. \& Scott D.W. 1993. The skin and appendages, p.531-738. In: Jubb K.V.F., Kennedy P.C. \& Palmer N.C. (Eds), Pathology of Domestic Animals. Academic Press, California. 